\title{
Nivel de información y conducta farmacoterapéutica de los odontólogos, 1990*
}

\author{
Levels of information and pharmaco-therapeutical behavior of dentists, 1990
}

\author{
Luis Jose Battellino**, Fernando Ruben Bennun**
}

BATTELLINO, L.J. \& BENNUN, F.R. Nivel de información y conducta farmacoterapética de los odontólogos. Rev. Saúde Pública, 27: 291-9, 1993 . Se describen los resultados de una investigación destinada a evaluat conocimientos farmacológicos, conducta de prescripción y propuestas para mejorar el empleo de medicamentos en 285 odontólogos de la Ciudad de Córdoba (Córdoba, Argentina). El estudio mostró que el 58,3\% de los odontólogos encuestados obtiene información en farmacología y terapéutica principalmente a través del material proveniente de los laboratorios farmacoquímicos. Paralelamente, alrededor de un tercio de los encuestados respondió incorrectamente o dijo no conocer la respuesta a preguntas vinculadas con la farmacoterapéutica de los antimicrobianos y analgésicos-antiinflamatorios de su preferente prescripción. Aunque el $63,5 \%$ de los odontólogos dijo tomar en consideración el listado modelo de medicamentos esenciales propuesto para el sistema de la seguridad social, muchas de las drogas indicadas de preferencia no están incluídas en el mismo. Para antimicrobianos y analgésicos-antiinflamatorios, las marcas indicadas con mayor frecuencia fueron las de más alto valor económico, pese a que el $66,5 \%$ de los encuestados afirmó orientar la prescripción hacia los productos de menor costo. La investigación reveló que cinco empresas farmaco-químicas concentran la provisión de antimicrobianos y analgésicos-antiinflamtorios prescriptos de preferencia por el $83,7 \%$ y $82,4 \%$, respectivamente, de los odontólogos consultados.

Descriptores: Odontólogos. Prescripción de medicamentos. Farmacología.

\section{Introducción}

Está fuera de discusión que los medicamentos de buena calidad desempeñan una importante función en la prevención y/o tratamiento de diversas enfermedades, así como en el alivio de muchos de sus síntomas. Por ello, el empleo óptimo y racional de los productos medicinales es una condición fundamental para el correcto ejercicio de la práctica profesional, además de tener importantes implicancias económicas.

Sin embargo, en muchos países del mundo en desarrollo, los medicamentos son empleados con frecuencia en forma irracional, lo cual acarrea dos tipos de consecuencias indeseables. Por un lado, exponen a quienes están bajo tratamiento farmacoterapéutico a riesgos indebidos. Por otra parte,

\footnotetext{
* Parte de trabajo de investigación subsidiado con una beca de perfeccionamiento otorgada al Dr Femando Rubén Bennún por la Secretaría de Extensión Universitaria de la Universidad Nacional de Córdoba, año 1990.

* Cátreda "B" de Química y Física Biológicas de la Facultad de Odontología, Universidad Nacional de Córdoba
}

Separatas/Reprints: L.J. Battellino - Pabellón Argentina - Ciudad Universitaria (Estafeta 32) (5000) Córdoba - Argentina. generan gastos superfluos de divisas a las personas, al sistema de la seguridad social y/o al Esta$\mathrm{do}^{2,10}$. Es de allí que las acciones tendientes a racionalizar el consumo de medicamentos tengan no sólo interés económico, sino también y especialmente un objetivo de protección de la salud comunitaria.

El problema del uso no racional de los medicamentos se fundamenta principalmente en la existencia de malas prácticas de prescripción por parte de los profesionales de la salud, lo que a su vez, en buena medida, radica en la deficiente capacitación que ellos poseen en las áreas farmacológica y terapéutica. La falta de conocimientos o la información imprecisa o equivocada acerca de las propiedades y usos de los medicamentos determina decisiones erróneas en el momento de la elección terapéutica.

Sobre estas falencias se asienta la intensa publicidad desplegada por las empresas farmacoquímicas, proporcionando información capaz de inducir la prescripción, el suministro o la adquisición de medicamentos. Con frecuencia, dicha información no reune los requisitos básicos de suficiencia, coherencia y precisión respecto a los fármacos cuya venta promueven los laboratorios fabricantes, necesarios para que la indicación, dispensación y el consumo de medicamentos re- 
sulten apropiados. A este modelo de promoción están expuestos los profesionales de la salud en Argentina, y probableminte también en el resto de países con subdesarrollo económico, y en ella confían firmemente en el momento de decidir la prescripción, sin reconocerla como uno de los factores capaces de provocar la indicación equivocada o excesiva de medicamentos. Queda completado así un círculo vicioso donde desinformación profesional/promoción comercial deficiente constituyen dos elementos en continua retroalimentación. El estudio realizado por Battellino ${ }^{3}$ en la población de médicos cordobeses confirma esta interacción.

Aunque en un campo mucho más restringido de la farmacoterapia, los odontólogos ejercen también la faculdad de prescribir medicamentos, particularmente aquéllos dotados de acción antimicrobiana $o$ analgésica-antiinflamatoria. Ante la inexistencia de estudios previos a nivel nacional, desarrollamos en 1990 una investigación epidemiológica en la población de odontólogos que realizan su prática profesional en la Ciudad de Córdoba (Provincia de Córdoba, Argentina), tendiente a evaluar sus conocimientos, hábitos y actitudes respecto a la utilización de medicamentos.

El presente trabajo describe y analiza los resultados de dicha investigación, cuyos objetivos fueran:

- Establecer el nivel de información farmacológica y los hábitos de prescripción de los odontólogos en lo referente a la calidad (elección de monodrogas, efectos colatcrales, contra indicaciones, tiempo de tratamiento, posibilidades de asociación) y al valor económico de la indicación terapéutica (por monodrogas, por marca registrada, por tratamiento completo).

- Conocer el diagnóstico de la situación y las propuestas que formulan los odontólogos para mejorar la eficiencia de la prescripción de medicamentos (implementación del Formulario Terapéutico Unico, prescripción de drogas por su nombre genérico, prohibición de la propaganda comercial en el rubro farmacoquímico, mejoramiento de nivel académico de grado y de postgrado, necesidad de modificar las pautas para autorizar la comercialización de productos medicinales).

\section{Material y Metodos}

\section{Población de estudio y tamaño muestral}

La investigación consistió en un estudio descriptivo transversal, realizado sobre la pobla- ción de odontólogos que ejercen actividad profesional en el ámbito de la Ciudad de Córdoba*, durante el período comprendido entre los meses de febrero y mayo de 1990. La muestra fue seleccionada por el procedimiento aleatorio simple, comprendiendo un total de 285 odontólogos que representaron el $28,3 \%$ de los profesionales habilitados a ese tiempo en la Ciudad de Córdoba. La muestra total fue dividida en varios subgrupos, de acuerdo a características de los profesionales relacionadas con el tiempo transcurrido desde el egreso de la carrera de grado (43,2\% con no más de 5 años de egreso y $56,8 \%$ con más de 5 años), tipo de inserción en el mercado laboral $(28,3 \%$ con actividad predominante en el subsector público, $50,7 \%$ en el privado y $21,0 \%$ en el sistema de la seguridad social) y formación de postgrado $(15,4 \%$ doctorados en odontologia y $84,6 \%$ sin graduación cuaternaria). Dado que la casi totalidad de los profesionales $(95,5 \%)$ egresaron de la Universidad $\mathrm{Na}$ cional de Córdoba, no se subdividió a la muestra por facultad de origen.

\section{Recolección de datos}

$\mathrm{El}$ instrumento para la recolección de datos consistió en una encuesta por cuestionario, conteniendo preguntas estructuradas y semiestructuradas $^{4}$. Previamente al desarrollo del cuestionario, los profesionales seleccionados en la muestra fueron entrevistados con el propósito de reiterar las instrucciones pertinentes al modo de responder cada una de las preguntas. El cuestionario fue respondido en forma anónima, en el tiempo que cada uno de los encuestados consideró necesario. Al receptar los cuestionarios se verificó que todas las preguntas hubieran sido respondidas completamente y según instrucciones, de modo que la tasa de respuestas válidas fue del $100 \%$. Cuando a una misma pregunta correspondieron dos o más respuestas no antagónicas, todas se tomaron en consideración y computadas como respuestas distintas.

\section{Evaluación estadistica}

Los datos fueron sometidos a tratamiento estadístico mediante el análisis de varianza ${ }^{22}$. El límite de significación estadística para establecer diferencias entre los subgrupos fue fijado en el valor $\mathrm{P}=0,05$.

\footnotetext{
* Según la Dirección de Estadísticas y Censo, la población de la Ciudad de Córdoba era en ese año de 1.140.135 habitantes, con una tasa de 1 odontólogo por cada 1.132 personas.
} 


\section{Resultados y Discusión}

Fuentes de información farmacoterapéuticas $y$ hábitos de prescripción de medicamentos

La Tabla 1 muestra que más de la mitad de los odontólogos encuestados $(58,3 \%)$ recurre para la obtención de información farmacológica y terapéutica principalmente a fuentes vinculadas a las empresas farmacoquímicas (índices de especialidades medicinales $45,2 \%$, literatura provista por los laboratorios $13,1 \%$ ). Como fuentes alternativas reconocieron su preferencia por los textos de farmacología y terapéutica, las actividades de postgrado destinadas a la actualización y las revistas propias de la especialidad, en ese orden de frecuencia. No se observaron diferencias estadisticamente significativas en la selección preferencial de la fuente de información según tiempo de egreso de los odontólogos ni del tipo de inserción en el mercado laboral. En cambio, hubo diferencias estadísticamente significativas $(P<0,01)$ en las respuestas según formación de postgrado: los profesionales doctorados en odontología mostraron mayor preferencia $(30,0 \%)$ que los que no tenían graduación cuaternaria $(2,8 \%)$ por las revistas propias de la especialidad, en tanto que estos últimos seleccionaron en mayor proporción que aquéllos los Indices de Especialidades Medicinales $(65,4 \%$ y $22,8 \%$, respectivamente).

Tabla 1. Fuentes de información farmacoterapéutica utilizadas por los profesionales odontólogos, Ciudad de Córdoba, año 1990 (en porcentaje del total de encuestados).

\begin{tabular}{lc}
\hline \multicolumn{1}{c}{ Fuente de información principal } & $\%$ \\
\hline $\begin{array}{l}\text { Indices de especialidades medicinales } \\
\text { (Vademecum Vallory y otros) }\end{array}$ & 45,2 \\
Textos de farmacologia y terapéutica & 18,2 \\
$\begin{array}{l}\text { Actividades científicas de postgrado } \\
\text { (jornadas, cursos, etc.) }\end{array}$ & 15,0 \\
$\begin{array}{l}\text { Literatura promocional provista por los } \\
\text { laboratorios farmacoquímicos }\end{array}$ & 13,0 \\
$\begin{array}{l}\text { Revistas especializadas de farmacologia } \\
\text { y terapéutica }\end{array}$ & 8,0 \\
\hline Total & 100,0 \\
\hline
\end{tabular}

Independientemente del tiempo de egreso, formación de postgrado e inserción laboral, casi dos tercios de los odontólogos encuestados dijo que al momento de prescribir tomaba en consideración siempre $(2,7 \%)$ o frecuentemente $(60,8 \%)$ el listado modelo de medicamentos contenidos en el Formulario Terapéutico Odontológico, elaborado para prestadores y beneficiarios del Instituto Pro- vincial de Atención Médica (IPAM), en base a eficacia y seguridad de los fármacos (Tabla 2). A su vez, una fracción correspondiente a alrededor de las dos terceras partes de la muestra dijo tener en cuenta siempre $(9,5 \%)$ o frecuentemente $(56,8 \%)$ el valor económico de los medicamentos al realizar la prescripción, de modo que a similar eficacia y seguridad preferían los productos de marca que tenían menor costo. Solamente por excepción $(1,3 \%)$ la indicación farmacoterápica se realizaba prescindiendo del valor comercial del producto prescripto. Casi la totalidad de los odontólogos afirmó que siempre $(59,5 \%)$ o frecuentemente $(33,7 \%)$ producían la prescripción en los recetarios provistos por las obras sociales, lo cual posibilitaba al usuario obtener financiación o descuento en la adquisición de medicamentos, aunque en su mayor parte aclararon que aplicaban este criterio sólo cuando el paciente portaba a la consulta el correspondiente recetario, sin realizar en la oportunidad ninguna acción educativa orientada a favorecer esa conducta.

Tabla 2. Habitos de prescripción de medicamentos por los profesionales odontólogos, Ciudad de Córdoba, año 1990.

\begin{tabular}{llllll}
\hline $\begin{array}{c}\text { Factor tomado en } \\
\text { cuenta al prescribir }\end{array}$ & $S$ & $F$ & $O$ & $N$ & Total \\
\hline
\end{tabular}

\begin{tabular}{|c|c|c|c|c|c|}
\hline $\begin{array}{l}\text { Listado modelo de } \\
\text { medicamentos } \\
\text { Valor económico del } \\
\text { producto } \\
\text { Recetario de la obra } \\
\text { social }\end{array}$ & $\begin{array}{r}2,7 \\
59,5 \\
9,5\end{array}$ & $\begin{array}{l}60,8 \\
33,7 \\
56,8\end{array}$ & 5,4 & 1,4 & $\begin{array}{l}100,0 \\
100,0 \\
100,0\end{array}$ \\
\hline $\begin{array}{l}S=\text { siempre } \\
O=\text { ocasionalmente }\end{array}$ & \multicolumn{5}{|c|}{$\begin{array}{l}F=\text { frecuentemente } \\
N=\text { nunca }\end{array}$} \\
\hline
\end{tabular}

Como lo indica la Tabla 3, los antibióticos Blactámicos fueron los antimicrobianos de preferencia en el tratamiento de los procesos infecciosos que se presentan en la práctica odontológica (ampicilina $32,1 \%$, penicilinas $23,0 \%$ y amoxicilina $22,8 \%$ ), lo cual es totalmente compatible con la sensibilidad que tienen habitualmente los gérmenes causales de cuadros sépticos frente a dichos fármacos. Como antibiótico de alternativa fue seleccionada la eritromicina, probablemente para resolver situaciones de hipersensibilidad o resistencia a las penicilinas. Tetraciclinas y cefalosporinas, en conjunto, fueron las de prescripción menos frecuentes. En todos los subgrupos de odontólogos se registraron respuestas similares en cuanto a la selección de agentes antimicrobianos, los cuales, en 
Tabla 3. Estructura de prescripción de antimicrobianos por profesionales odontólogos, Ciudad de Córdoba, año 1990.

\begin{tabular}{|c|c|c|c|c|}
\hline Monodrogas & Elección* & $\begin{array}{l}\text { Productos } \\
\text { comerciales }\end{array}$ & $\begin{array}{l}\text { Marcas } \\
\text { registradas }\end{array}$ & $\begin{array}{l}\text { Costo relativo por } \\
\text { tratamiento }\end{array}$ \\
\hline Ampicilina & 32,1 & 34,9 & $\begin{array}{l}a=63,2 \\
b=31,6 \\
c=3,1 \\
d=2,1\end{array}$ & $\begin{array}{r}100 \\
51 \\
28 \\
52\end{array}$ \\
\hline Penicilinas & 23,0 & 12,0 & $\begin{array}{l}\theta=96,9 \\
f=3,1 \\
g=0,0\end{array}$ & $\begin{array}{r}100 \\
64 \\
32\end{array}$ \\
\hline Amoxicilina & 22,8 & 26,7 & $\begin{aligned} h & =50,0 \\
i & =44,9 \\
& =5,1 \\
k & =0,0\end{aligned}$ & $\begin{array}{r}100 \\
91 \\
72 \\
58\end{array}$ \\
\hline Eritromicina & 15,8 & 17,8 & $\begin{array}{l}\mathrm{l}=61,7 \\
\mathrm{~m}=38,3 \\
\mathrm{n}=0,0\end{array}$ & $\begin{array}{r}100 \\
90 \\
81\end{array}$ \\
\hline Tetraciclinas & 4,3 & 4,7 & $\begin{array}{l}0=53,9 \\
p=46,1\end{array}$ & $\begin{array}{r}100 \\
34\end{array}$ \\
\hline Cefalosporinas & 2,0 & 3,9 & $\begin{array}{l}q=100,0 \\
r=0,0\end{array}$ & $\begin{array}{r}100 \\
63\end{array}$ \\
\hline
\end{tabular}

- Indica el porcentaje de encuestados que ubicó a las respectivas monodrogas en el primer lugar de elección.

* Indica el porcentaje de encuestados que prescribió alguno de los productos comerciales existentes en plaza, conteniendo la respectiva monodroga seleccionada.

*** Indica la frecuencia porcentual de encuestados que prescribe un producto de marca registrada (a, b, etc) correspondiente a la monodroga seleccionada.

**** Representa los valores relativos respecto al producto comercial de mayor precio $(=100)$ que contiene la monodroga seleccionada, para todo el período de tratamiento, incluyendo las unidades excedentes, al mes de julio de 1990.

Además de las monodrogas alistadas en el cuadro, se incluyeron en la encuesta antimicrobianos como gentamicina, kanamicina, ácido nalidíxico, sulfametoxazol-trimetoprima y otros, que en ningún caso fueron seleccionados como drogas de preferencia.

su mayoría, están contenidos en el listado modelo del Formulario Terapéutico Odontológico.

En la forma de prescribir en base a nombres de fantasía, la elección se inclinó a favor de marcas registradas compuestas por ampicilina $(34,9 \%)$, amoxicilina $(28,7 \%)$ y eritromicina $(17,3 \%)$. Estas preferencias tienen buena correspondencia con las elecciones efectuadas de acuerdo al fármaco activo, de manera que la relación monodrogas/ productos comerciales seleccionados fue muy cercana a la unidad para esos antibióticos. No ocurrió lo propio respecto a las penicilinas y cefalosporinas, donde la relación prácticamente se duplicó para las primeras y se redujo a la mitad para las últimas. Esta falta de correspondencia fue proporcionalmente más alta en los profesionales sin graduación cuartenaria que en los doctorados en odontología.

Al analizar la prescripción de antibióticos en relación a su valor de venta, surge que en el caso de las ampicilinas el producto más recetado es el de mayor precio (marca $a=100$ ), siendo su valor comercial casi el doble del que tiene el producto que le sigue en preferencia (marca $b=51$ ). Una situación similar se planteó en cuanto a las preparaciones comerciales que contienen penicilinas y amoxicilina, donde los productos preferentemente escogidos (marcas e y h, respectivamente) son los más costosos, ya sea por unidad o por tratamiento completo.

En cuanto a la elección de analgésicosantiinflamatorios, el piroxicam y el naproxeno representaron las monodrogas predilectas para casi dos tercios $(36,1 \%$ y $29,8 \%$, respectivamente) de los odontólogos encuestados (Tabla 4), sin que existieran diferencias significativas en las respuestas formuladas por los distintos subgrupos de odontólogos. El resto repartió sus preferencias entre otras siete monodrogas, incluidas dipirona y paracetamol, que tienen aplicación preferente- 
Tabla 4. Estructura de prescripción de analgésicos antiinflamatorios por profesionales odontólogos, Ciudad de Córdoba, año 1990.

\begin{tabular}{|c|c|c|c|c|}
\hline Monodrogas & Elección* & $\begin{array}{l}\text { Productos } \\
\text { comerciales** }\end{array}$ & $\begin{array}{l}\text { Marcas } \\
\text { registradas }\end{array}$ & $\begin{array}{l}\text { Costo relativo por } \\
\text { tratamiento }\end{array}$ \\
\hline Piroxicam & 36,1 & 44,5 & $\begin{array}{l}a^{\prime}=46,1 \\
b^{\prime}=42,7 \\
c^{\prime}=6,8 \\
d^{\prime}=4,4\end{array}$ & $\begin{array}{r}100 \\
25 \\
70 \\
46\end{array}$ \\
\hline Naproxeno & 29,8 & 15,4 & $\begin{array}{l}e^{\prime}=88,7 \\
f^{\prime}=11,3\end{array}$ & $\begin{array}{r}100 \\
40\end{array}$ \\
\hline \multicolumn{5}{|l|}{ Acido } \\
\hline Mefenamico & 9,1 & 9,1 & $g^{\prime}=100,0$ & 100 \\
\hline Dipirona & 7,2 & 10,5 & $\begin{aligned} h^{\prime}= & 45,0 \\
i^{\prime}= & 30,0 \\
j^{\prime} & =22,5 \\
k^{\prime}= & 2,5\end{aligned}$ & $\begin{array}{r}68 \\
92 \\
73 \\
100\end{array}$ \\
\hline Aspirina & 5,3 & 5,5 & $I^{\prime}=100,0$ & 100 \\
\hline Indometacina & 5,3 & 5,8 & $\begin{array}{l}m^{\prime}=88,2 \\
n^{\prime}=11,8\end{array}$ & $\begin{array}{r}100 \\
75\end{array}$ \\
\hline Ibuprofeno & 4,1 & 4,3 & $0^{\circ}=100,0$ & 100 \\
\hline Paracetamol & 1,8 & 3,6 & $\begin{array}{l}p^{\prime}=66,6 \\
q^{\prime}=33,4\end{array}$ & $\begin{array}{r}57 \\
100\end{array}$ \\
\hline Ketoprofeno & 1,2 & 1,3 & $r^{\prime}=100,0$ & 100 \\
\hline
\end{tabular}

- Indica el porcentaje de encuestados que ubicó a las respectivas monodrogas en el primer lugar de elección.

* Indica el porcentaje de encuestados que prescribió alguno de los productos comerciales existentes en plaza, conteniendo la respectiva monodroga seleccionada.

*** Representa la frecuencia porcentual de encuestados que prescribía un producto de marca registrada (a', b', elc) correspondiente a la monodroga seleccionada.

*** Representa los valores relativos respecto al producto comercial de mayor precio $(=100)$ que contiene la monodroga seleccionada, para todo el período de tratamiento, incluyendo las unidades excedentes, al mes de julio de 1990.

Además de las monodrogas alistadas en el cuadro, se incluyeron en la encuesta analgésicos antiinflamatorios como diclofenac, sulindac, tolmetina, ácido flufenamico y diflunisal, los que en ningún caso fueron seleccionados como drogas de preferencia.

mente por su actividad antipirética. La prescripción de productos de marca conteniendo piroxicam $(44,5 \%)$ fue mayor que la correspondiente a monodrogas, ocurriendo lo contrario en el caso de productos preparados en base a naproxeno $(15,4 \%)$. Exceptuadas dipirona y paracetamol, en las restantes monodrogas de esta clase terapéutica los productos de marca de mayor prescripción fueron los de costo más alto, por unidad y por tratamiento.

Nivel de información disponible respecto a los fármacos antimicrobianos y analgésicosantiinflamatorios

Alrededor de dos tercios de los profesionales encuestados respondió correctamente a las preguntas relacionadas con algunos aspectos farma- cológicos, terapéuticos y toxicológicos de los antimicrobianos y analgésicos-antiinflamatorios no esteroideos, en tanto el resto lo hizo erróneamente o dijo desconocer el mecanismo de acción (41,5\%), posibilidad de empleo durante el embarazo $(31,0 \%)$, duración habitual del tratamiento $(33,0 \%)$, conveniencia de utilizarlos asociados con otras drogas de la misma clase terapéutica $(41,0 \%)$ y presentación de efectos colaterales $(29,5 \%)$ (Tabla 5). La tasa de respuestas correctas fue significativamente mayor $(\mathrm{P}<0,01)$ en los doctorados en odontología $(85,5 \%)$ que en los profesionales sin graduación cuaternaria $(40,4 \%)$. El $92,0 \%$ de las preguntas en las que se dijo desconocer las respuestas 0 que las mismas resultaron incorrectas correspondieron a odontólogos cuya fuente principal de información provenía de laboratorios farmacoquímicos. 
Tabla 5. Nivel de conocimientos farcoterapéuticos de los profesionales odontólogos sobre drogas antimicrobianas y analgésicas antiinflamatorias, Ciudad de Córdoba, año 1990. (En porcentaje del total de encuestados)

\begin{tabular}{lccccc}
\hline \multicolumn{1}{c}{$\begin{array}{c}\text { Aspecto } \\
\text { evaluado }\end{array}$} & $\begin{array}{c}\text { Respondió } \\
\text { bien }\end{array}$ & $\begin{array}{c}\text { Respondió } \\
\text { mal }\end{array}$ & $\begin{array}{c}\text { No } \\
\text { sabe }\end{array}$ & Total \\
\hline $\begin{array}{l}\text { Mecanismo de } \\
\text { acción }\end{array}$ & 58,5 & 22,5 & 19,0 & 100,0 \\
$\begin{array}{l}\text { Empleo durante el } \\
\text { embarazo }\end{array}$ & 69,0 & 18,0 & 13,0 & 100,0 \\
$\begin{array}{l}\text { Duración del } \\
\text { tratamiento }\end{array}$ & 67,0 & 33,0 & 0,0 & 100,0 \\
$\begin{array}{l}\text { Conveniencia de } \\
\text { asociaciones }\end{array}$ & 59,0 & 21,0 & 20,0 & 100,0 \\
$\begin{array}{l}\text { Efectos adversos } \\
\text { y/o colaterales }\end{array}$ & 70,5 & 20,5 & 9,0 & 100,0 \\
\hline
\end{tabular}

Estructura de la prescripción de antimicrobianos y analgésicos-antiinflamatorios de marca registrada, segün laboratoriofabricante

Como lo muestra la Figura, existe un alto grado de concentración en cuanto a la procedencia de los productos de marca preferentemente prescriptos en la terapia antimicrobiana y analgésicaantiinflamatoria. En ambos casos, un mismo laboratorio (A) produce tanto los antimicrobianos como los analgésicos-antiinflamatorios indicados de preferencia por alrededor de la tercera parte de los odontólogos consultados $(34,7 \%$ y $33,0 \%$, respectivamente). En el rubro de los antimicrobianos, cinco empresas farmacoquímicas proveen las especialidades medicinales preferentemente prescriptas por el $83,7 \%$ de los odontólogos, en tanto que igual número de laboratorios fabricantes comercializan los productos de marca de analgésicosantiinflamatorios elegidos para la indicación por el $82,4 \%$ de los profesionales. Solamente una de dichas empresas (A) tiene participación casi por igual en el mercado local de comercialización de antimicrobianos y analgésicos-antiinflamatorios de uso odontológico.

Medidas sugeridas para mejorar la calidad de la prescripción farmacológica en odontología

Los odontólogos encuestados distribuyeron con relativa paridad sus preferencias en la selección de las alternativas formuladas en la encuesta: mejoramiento del nivel académico en la carrera odontológica $(26,0 \%)$, control de calidad, eficacia y seguridad de los medicamentos por parte de los organismos de salud pública $(23,7 \%)$, implementación de un Formulario Terapéutico Unico de uso obligatorio $(19,5 \%)$, prescripción de medicamentos por su nombre genérico $(19,2 \%)$ y prohibición de la propaganda comercial sobre medicamentos $(11,6 \%)$. Requeridos respecto a la comercialización en nuestro país de medicamentos no autorizados en paises de mayor desarrollo industrial, los profesionales odontólogos reconocieron como causas principales de dicha discordancia a las siguientes: incapacidad de control por los organismos sanitarios nacionales $(38,4 \%)$, presiones comerciales para su autorización $(29,0 \%)$ y existencia de normas demasiado permisivas en el ordenamiento legal argentino $(15,0 \%)$.

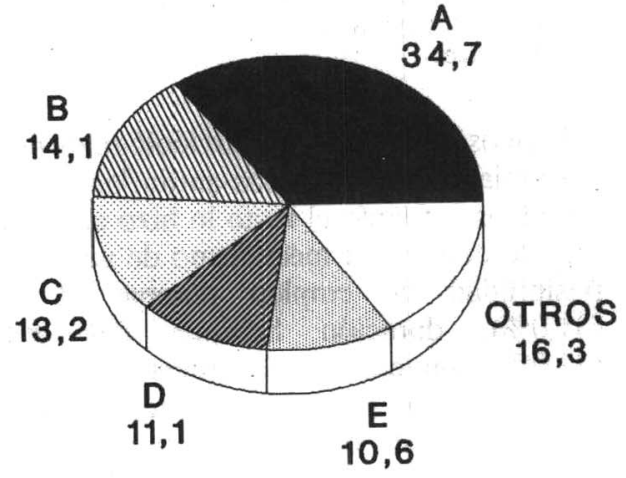

Antimicrobianos

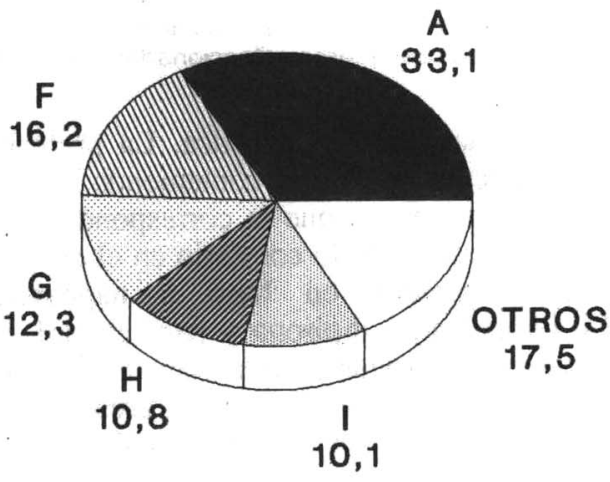

Analgésicos-antiinflamatorios

Las letras (A, B, C, etc.) identifican a los distintos laboratorios farmacoquímicos encargados de la elaboración de antimicrobianos y/o analgésicos antiinflamatorios de marca registrada.

Los valores numéricos colocados debajo de cada letra indican el porcentaje de odontólogos que prescriben de preferencia los antimicrobianos (izquierda) y los analgésicos antiinflamatorios (derecha) provistos por los correspondientes fabricantes.

Figura. Procedencia de los antimicrobianos y analgésicos-antiinflamatorios de marca registrada prescriptos por los profesionales odontólogos, Ciudad de Córdoba, año 1990. 
De la investigación que aquí se describe surge principalmente que sólo una baja proporción de los odontólogos que ejercen su actividad profesional en la Ciudad de Córdoba obtienen información farmacoterapéutica de fuentes independientes de la industria farmacéutica, que es deficiente su nivel de capacitación en cuanto a las propiedades, indicaciones, modo de empleo y efectos colaterales derivados de la aplicación de antimicrobianos y analgésicos-antiinflamatorios, y que la comercialización de productos de marca registrada conteniendo dichas clases de drogas en el sector odontológico está concentrada a favor de un reducido número de empresas fabricantes y/o distribuidoras de medicamentos. Es probable que tales características respondan a un denominador común, vinculado fundamentalmente a la actividad de promoción desarrollada por los laboratorios farmacoquímicos de mayor capacidad operativa, y que, en coincidencia con la opinión de los odontólogos encuestados, deberían ser resueltas a partir de la formulación e implementación de políticas originadas en los organismos centrales de la conducción sanitaria nacional.

Por las consecuencias médicas, sociales y económicas resultantes, la prescripción y dispensación de medicamentos debe estar basada en sólidos conocimientos científicos, de modo de favorecer la obtención de los mejores resultados con el mínimo de efectos nocivos y al costo más bajo posible. Sin dudas, la industria farmacoquímica proporciona actualmente la mayor parte de la información sobre medicamentos a los profesionales de la salud. Desgraciadamente, y en palabras de Herxheimer, "... la información de los fabricantes de medicamentos suele ser parcial respecto de los fármacos cuya venta promueve, y a menudo los intereses de la promoción están en conflicto con la difusión de informaciones imparciales ${ }^{9 "}$. De acuerdo con Silverman ${ }^{17}$, las compañías farmacéuticas multinacionales utilizan diferentes mensajes para describir medicamentos que contienen el mismo principio activo según se trate de países industrializados o de naciones del Tercer Mundo. Para aquéllos está exigida una declaración completa de los efectos colaterales desagradables, peligrosos o potencialmente letales. En estas últimas, "las indicaciones son mucho más extensas, pero la enumeración de los riesgos se abrevia, se restringe o se omite totalmente. En algunos casos, sólo se describen los efectos colaterales triviales, en tanto las reacciones serias o posiblemente fatales no son mencionadas $17^{\prime \prime}$.

Pese a que la Federación Internacional de la Industria del Medicamer.to (FIIM) exige que la información farmacoterapéutica esté basada en una evaluación actualizada de todos los indicios científicos disponibles, en su mayor parte se trata de un material de mala calidad. Esta caracterización es aplicable a la información que se distribuye tanto a nivel nacional ${ }^{3}$ como en otros países ${ }^{12,17}$, incluyendo los Estados Unidos de Norte América, donde la Administración de Alimentos y Drogas (FDA) reveló que la gran mayoría del material de promoción presentado para su examen por parte de los fabricantes es falso y/o equivoco ${ }^{7}$. Por las omisiones, generalizaciones e inexactitudes en las que incurre, así como por sus falsedades y afirmaciones inadmisibles, el $72,0 \%$ de la información correspondiente a los productos de marca registrada incluidos en los Indices de Especialidades Medicinales de circulación en Argentina es de regular a baja calidad (4). Similar juicio han merecido referencias farmacoterapéuticas difundidas en otros países ${ }^{6,18}$.

El bajo nivel de conocimientos en el área de la farmacoterapéutica de incumbencia habitual de los odontólogos (tratamiento del dolor-inflamacióninfección del sistema estomatognático) quedó expresado no sólo en la desinformación en cuanto al mecanismo de acción, duración del tratamiento, posibilidades de asociación, efectos colaterales y contraindicaciones, sino también en el criterio de elección de monodrogas y en la falta de correspondencia entre fármacos seleccionados y productos de marca de prescripción más frecuente. Así es como el piroxicam se constituyó en el analgésico-antiinflamatorio de preferencia para más de un tercio de los encuestados, siguiéndole en orden de frecuencia el naproxeno. Ambas drogas superaron en alrededor de siete veces la preferencia por aspirina, sin que existan fundamentos valederos que justifiquen esas opciones. Diversos estudios clínicos controlados han demostrado que la actividad analgésica del piroxicam en el tratamiento de las odontalgias es aproximadamente la misma $^{19,21}$ o un poco mayor ${ }^{14,15,20}$ que la de aspirina, si bien tales diferencias tienen escasa significación clínica. Por otra parte, tomando en consideración parámetros tales como eficacia, inocuidad, costo de un plan de tratamiento, observancia, uso múltiple, facilidad de administración y disponibilidad, Mathur et al. ${ }^{11}$ seleccionaron recientemente a la aspirina como el analgésicoantiinflamatorio de mayor valor terapéutico. Por último, piroxicam no está incluído en la Sexta Lista Modelo de Medicamentos Esenciales propuesta por el Comité de Expertos de la Organización Mundial de la Salud ${ }^{5}$, ni su uso ha sido recomendado por la Convención de la Farmacopea de los Estados Unidos de América en las directrices para la prescripción odontológica ${ }^{13}$.

Existe concenso de que el empleo racional de medicamentos comprende la elección de acuerdo 
con las prioridades de la salud, el valor terapéutico, la inocuidad, la disponibilidad y la aceptación por parte del paciente, así como también un riguroso planteamiento económico a fin de utilizar los recursos del modo más ventajoso y adaptarlos a la situación socio-económica vigente ${ }^{16}$. De acuerdo a este último criterio, se concede particular interés a la prescripción eficaz respecto al costo ${ }^{1}$. No obstante que en su mayoría los odontólogos de la Ciudad de Córdoba afirmaron tomar en consideración el valor económico al seleccionar el medicamento adecuado, sus opciones por los productos de marca registrada de más alto costo mostraron lo contrario, sin que existan evidencias que sugieran diferencias de calidad a favor de las marcas más prescriptas. Probablemente ello ha generado distorsiones en la cadena de suministro de medicamentos, impidiendo a los grupos socioeconómicos más vulnerables satisfacer sus necesidades en salud, incluso las fundamentales.

En principio, tanto médicos como odontólogos tienen la opción de elegir libremente el medicamento más adecuado a los fines de resolver el motivo de la consulta, pero en la práctica su prescripción está condicionada a la presión de una publicidad que 10 induce a recetar determinados productos de marca. Por ello no es de extrañar que de las veinticinco preparaciones comerciales de piroxicam autorizadas para la comercialización en nuestro país, los odontólogos inclinen su preferencia hacia el producto que es elaborado y distribuido por el laboratorio (A) que mayor promoción desarrolla a nivel de esa profesión biomédica. Algo similar ocurre con naproxeno, ampicilina y amoxicilina. De esta forma, un reducido número de empresas farmacoquímicas logra concentrar la comercialización de los grupos de drogas de empleo más frecuente en la profesión odontológica, lo cual es un indicador de la distribución oligopólica del mercado farmacéutico nacional ${ }^{8}$.

Si bien es incuestionable que el nivel de salud de las personas no depende primariamente del sector sanitario, no es menos cierto que la calidad de la atención brindada por dicho sector puede favorecer o disminuir los efectos producidos por diversos factores que condicionan su existencia. De ello deriva la necesidad de elaborar y poner en práctica políticas sanitarias que posibiliten a todas las personas el acceso oportuno a las prestaciones de la más alta calidad disponible, conforme a sus necesidades. En dichas políticas, los medicamentos constituyen un componente crítico. A las universidades corresponde no sólo la función de interesar a otros sectores en la definición de esas políticas, sino el liderazgo en la capacitación del recurso humano profesional y técnico vinculado con las tareas de investigación, produc- ción, control, prescripción y evaluación de los medicamentos. Ésta será una valiosa contribución de las universidades a los fines de alcanzar la meta de Salud Para Todos en el Año 2000.

BATTELLINO, L.J. \& BENNUN, F.R. Nível de informação e conduta farmaco-terapêutica dos odontólogos, 1990. Rev. Saúde Pública, 27: 291-9, 1993. Descrevemse os resultados de pesquisa destinada a avaliar conhecimentos farmacológicos, conducta de prescrição e propostas para melhorar o emprego de medicamentos em 285 odontólogos da Cidade de Córdoba (Córdoba, Argentina). $O$ estudo mostrou que $58,3 \%$ dos pesquisados obtêm informação em farmacologia e terapêutica principalmente através do material que provém dos laboratórios farmacoquímicos. Paralelamente, por volta de um terço dos pesquisados respondeu incorretamente ou não respondeu perguntas ligadas com a farmacoterapêutica dos antimicrobianos e analgésicos antiinflamatórios de sua prescrição preferida. Embora $63,5 \%$ dos odontólogos dissessem levar em consideração a lista de medicamentos essenciais proposto para o sistema da segurança social, muitas das drogas indicadas como de preferência não estão incluídas na mesma. Para antimicrobianos e analgésicos anti-inflamatórios as marcas indicadas com maior frequência foram as de maior valor econômico, se bem que $66,5 \%$ dos indagados afirmaram orientar a prescrição para os produtos de menor custo. A pesquisa revelou que cinco empresas farmacoquímicas concentram a provisão de antimicrobianos e analgésicos anti-inflamatórios prescritos da preferência respectivamente de $83,7 \%$ e $82,4 \%$ dos odontólogos consultados.

Descritores: Odontólogos. Prescrição de medicamentos. Farmacologia.

BATTELLINO, L.J. \& BENNUN, F.R. [Levels of information and pharmaco-therapeutical behavior of dentists, 1990]. Rev. Saúde Pública, 27: 291-9, 1993. Resilts of an investigation carried out into a population of 285 dentists in Cordoba City (Cordoba, Argentine) with a view to evaluating their pharmacological knowledge, presstiptive behaviour and proposals for the improvement of cirug usage, are described. This study showed that $58.3 \%$ of the dentists surveyed get most of their pharmacological and therapeutic information through published material issued by pharmacochemical labs. At the same time, about one-third of the individuals surveyed either did not answer or gave incorrect answers to questions related to the pharmacotherapeutic effects the antimicrobial or anti-inflammatory analgesics of their preference. Although $63.5 \%$ of the dentists said that they had taken the essential drug model reference list issued by the social security system into account, many of the drugs selected as preferences were not to be found on it. Regarding anti-microbial or anti-inflammatory analgesic drugs, the brands most frequently prescribed were the more expensive ones, strongh $66.5 \%$ of the 
dentists surveyed stated the contrary. Finally, this investigation revealed that five manufacturers together held a concentration of the anti-microbial and antiinflammatory analgesics prescribed by $83.7 \%$ and $82.4 \%$ of the dentists consulted respectively.

Keywords: Dentists. Prescriptions, drug. Pharmacology.

\section{Referencias Bibliográficas}

1. AKHTAR, M.A. Rational therapeutics: a cost-effective approach. Clin. Pharmacol. Ther., 46: 164-72, 1990.

2. BATTELLINO, L.J. Eficiencia y seguridad de los medicamentos. Salud Soc., 1: 27-37, 1983.

3. BATTELLINO, L.J. La información farmacológica y la actividad médica. Cuad.Med. Soc., Rosario, 34:25-43, 1985.

4. CANALES, F.H. de et al. Diseño metodológico. In: Metodología de la investigación: manual para el desarrollo de personal de salud. México, Editorial Limusa, 1986. p. 165-71. (Serie Paltex $n^{9} 9$ ).

5. COMTTE DE EXPERTOS DE LA OMS EN USO DE MEDICAMENTOS ESENCIALES, Ginebra, 1989. Informe. Ginebra, Organización Mundial de la Salud, 1990. (OMS-, Serie de Informes Técnicos, 796).

6. ERILL, S. Clinical pharmacology in Spain. Clin. Pharmacol. Ther., 16: 597-604, 1974.

7. FDA's drugs problems. Scrip, (1.389): 14, 1989.

8. FERRARA, F. Los medicamentos en la Argentina: un enfoque global. Cuad. Med. Soc., Rosario, 22: 23-45, 1982.

9. HERXHEIMER, L.W. Normas coherentes en materia de medicamentos. Foro Mundial Salud, 3: 328-40, 1982.

10. KATZ, J. et al. Organización y comportamiento de los mercados prestadores de servicios de salud: reflexiones sobre el caso argentino. Buenos Aires, OPS, 1988. (Publicación $n^{2}$ ).
11. MATHUR, V.S. et al. Sistema de notación para la selección de medicamentos esenciales. Bol. Med. Esenc., 9: 19, 1990.

12. MEDAWAR, C. Insult or injury? An inquire into marketing and adversiting of British food and drug products in the Third World. London, Social Audit, 1979.

13. MINISTERIO DE SANIDAD Y CONSUMO DE ESPANAA/ ORGANTZACTON PANAMERICANA DE LA SALUD. Información de medicamentos. Madrid, 1989. 2 v. (OPAS - Publicación cientifica, 525).

14. NELSON, S.L. et al. Double-blind study of piroxicam (2 doses), aspirin and placebo in patients with postoperative dental pain. Clin. Pharmacol. Ther., 41: 182-95, 1987.

15. OLSON, N. et al. Piroxicam, aspirin and placebo in dental pain. Clin. Pharmacol. Ther., 41: 162-72, 1987.

16. ORGANIZACION MUNDIAL DE LA SALUD. Financiación de los medicamentos esenciales. Bol. Med. Esenc., 10: 12-3, 1990.

17. SILVERMAN, M. Epidemiología de la promoción del medicamento. Cuad. Med. Soc., Rosario, 19: 53-69, 1985.

18. STIMSON, G.V. Information contained on drug advertisements. Br. Med. J., 4: 508-13, 1975.

19. STRAVINKI, S. Piroxicam analgesis. Advance in Arthritis Therapy. April, 1982.

20. SUNSHINE, A. et al. The analgesic efficacy of piroxicam, aspirin and placebo in postoperative oral surgery pain. Clin. Pharmacol. Ther., 37: 232-9, 1985.

21. SUNSHINE, A. et al. Analgesic efficacy of piroxicam in the treatment of postoperative pain. Am.J. Med., 84 (Suppl. 5A): 16-22, 1988.

22. WINTRAUB, J.A. et al. Bioestadística en salud bucoden. tal. Washington, Organización Panamericana de la Salud, 1985.

Recebido para publicação em 26.11.1992 Reapresentado em 20.5.1993 Aprovado para publicação em 24.6.1993 